Novembre 2012 | www.ocula.it - DOI: 10.12977/ocula57

\title{
Monument et espace urbain Pour une Sémiotique des parcours et des structures de la ville
}

di Ruggero Ragonese

Università di Milano ruggero.ragonese@unimi.it

«Il faut des monuments aux cités de l'homme, autrement où serait la différence entre la ville et la fourmilière?»

Victor Hugo, Littérature et philosophie mêlées (1834), 1830

\begin{abstract}
Durant les 30 dernières années, l'approche de la sémiotique à l'architecture et à l'espace a souvent été ambiguë ou inefficace. Ambiguë parce que, la plupart du temps, on a cherché à regarder l'espace «représenté» à l'intérieur de systèmes sémiotiques déterminés (visuels et auditifs) et non l'espace concret du monde naturel. Inefficace parce que souvent, l'analyse des textes architecturaux n'a pas réussi à se détacher des limites imposées par la sémiotiques des codes des années ‘6o.

La sémiotique textuelle dans les années 80 e 90 a toujours abordé le problème avec embarras. Dans quelques rares cas la recherche a réussi à fournir des instruments aptes à l'analyse d'un texte spatial (parmi ces cas, on peut rappeler le numéro 73/4 de VS sur l'espace édité par Sandra Cavicchioli et les études de Renier).

Cependant, partant de cet exigu corpus bibliographique, on peut chercher des bases pour commencer un travail sur le texte architectural, à partir de l'étude de monuments, capable de décrire le processus de transformation urbaine et les formes de l'espace construit.
\end{abstract}

\section{Mots Clés}

Ville, paysage urbain, espace, monument, parcours

\section{Index}

1. L'espace du monument

2. Monumentaliser la ville

3. Petite conclusion sur semiotique et architecture

Bibliographié 


\section{Dcular Flux_saggi}

Ruggero Ragonese | Monument et espace urbain

\section{L'espace du Monument}

Entité complexe, le monument est non seulement un lieu commémoratif ou votif, mais aussi un carrefour textuel dont le caractère de la ville s'élabore symboliquement dans nombreux discours et parcours. Discours et parcours dévoilent et ouvrent le texte monumental dans la ville vers une analyse plus attentive et précise qui révèle les articulations profondes qui le composent. La propos développé dans cet contribution n'est pas de traiter des monuments comme 'édifices remarquables', mais de nous référer principalement aux monuments comme lieux de vie sociale de la ville. Le monument produit de la communauté, en désignant à elle-même comme force d'adhésion et de projet, à travers le partage de rituels et d'affects.

Où peut résider une analyse sémiotique de l'espace de la ville qui ne veut s'appesantir ni sur l'analyse historique ni sur celle architecturale? Ce travail devrait se diviser en trois parties. La première tendrait à déterminer un corpus de textes architecturaux dans la ville. Une fois le corpus déterminé, il s'agirait d'identifier à l'aide de textes littéraires qui les ont décrits, les parcours qui se sont succédés historiquement à l'intérieur de ces espaces. La dernière partie du travail consisterait (en réunissant dans ce cas-là une synchronique de l'architecture et une diachronique) à vérifier, à l'aide des instruments de la sémiotique plastique, les propres structures du texte architectural, déjà observé à travers ses parcours. D'un côté le monument architectural, comment a-t-il été et comment est-il vécu, de l'autre le texte monument. Ces parcours dévoilent et ouvrent le texte architectural dans la ville vers une analyse plus attentive et précise qui révèle les articulations profondes qui le composent.

Ainsi, Avant de nous demander ce qu'est un monument à l'intérieur d'un espace urbain, commençons par nous demander: quel type d'espace est le monument à l'intérieur du milieu urbain? De Sessa, à propos de l'espace monumental, nous donne une longue définition d'espace fermé dans son œuvre Capire lo spazio architettonico (1990), qui peut nous aider:

(N.d.T.) «comprendre l'espace fermé signifie laisser à l'extérieur la palpitation inquiète de la quotidienneté pour se raidir en plusieurs formes séparées; on abdique aux possibilités d'échange de la communication et surtout à ses caractéristiques dialogiques. On choisit un langage qui peut être, à chaque fois, redondant ou décharné, pompeux, raide, monumental, mais toujours intransigeant; en effet le concept d'espace en question ne produit pas de discours, mais bien au contraire des sermons; il utilise une construction rhétorique, utilisant des banalités et des expressions toutes faites afin de convaincre l'observateur sur ce qui a été affirmé.»1

1 De Sessa (1990), p. 207. «Cifra dello spazio chiuso è l'estromettere il palpito inquieto della quotidianità per irrigidirsi in forme separate, si abdica dalle possibilità di scambio della comunicazione e soprattutto, dalle caratteristiche dialogiche di questa. Si opta per un linguaggio che può essere, di volta in volta, ridondante o scarno, pomposo, rigido, monumentale, ma sempre intransigente; in effetti la spazialità in esame non produce discorsi bensì sermoni; si avvale di un periodare retorico, usando banalità e frasi fatte onde persuadere il fruitore circa la giustezza di quanto affermato.» 


\section{Oculartux_saggi}

Ruggero Ragonese | Monument et espace urbain

Parmi ces espaces fermés De Sessa cite quelques exemples: Le Paris de Haussman, l'U.R.S.S. de Stalin et le Vittoriano (ou autel de la Patrie, dit vulgairement la Machine à écrire) à Rome. On remarque très vite que, si

dans les deux premiers cas on cite deux exemples de politique urbaniste, la troisième est celui d'un seul et unique monument: le Vittoriano. Sur ce dernier, De Sessa donne un jugement à la fois sévère et plein d'ironie.

(N.d.T.) «Le Vittoriano, non seulement représente l'espace fermé le plus grotesque que l'on peut imaginer, mais il est une absurdité; passée, malgré la masse cyclopéenne mais surtout totalement inaccessible.»²

À notre avis, deux caractéristiques peuvent être appliquées à la monumentalité, si l'on suit le discours de De Sessa: elle est un espace fermé parce que séparée et inaccessible. Mais procédons par ordre et prenons un exemple: L'autel de la Patrie est-il un espace fermé? La définition d"espace fermé' donné peut nous sembler un peu faible. Si par espace fermé, on entend un espace inaccessible, on ne comprend pas comment insérer l'autel de la Patrie dans cette catégorie. L'autel de la patrie est absolument accessible, bien plus accessible qu'un lieu que l'on classifie comme espace 'ouvert', c'està-dire l'Acropole d'Athènes. Avant la fermeture du monument (en 1968), le sujet visiteur accomplissait presque toujours son parcours à l'intérieur du monument.

L'Autel de la patrie a comme élément caractéristique et conducteur, tout au moins jusqu'à l'arrivée des propylées, le grand escalier. Le premier grand escalier conduit à la tombe du soldat inconnu, les autres amènent tout d'abord aux deux grands portails du musée du Risorgimento et des drapeaux et ensuite à la statue de Vittorio Emanuele II; deux autres escaliers conduisent à la grande esplanade face aux propylées et enfin deux derniers qui amènent aux propylées de gauche et de droite. L'escalier est l'élément qui peut instaurer une isotopie spatiale à l'intérieur du monument. Il est évident que l'escalier, que l'on peut parcourir contient sur le plan du contenu, et à un niveau linguistique, le sème 'accessibilité'.

L'Autel veut donc être 'accessible' à tout le monde. On ne sait pas comment on peut définir un espace fermé, un monument qui présente un seul mur: celui qui soutient la haute colonnade, un mur qui entre parenthèses n'empêche pas au visiteur l'accès au panorama en-dessous de la colonnade, mais le contraint tout simplement à effectuer un plus grand tour qui l'oblige à passer sous un des deux propylées.

Ibidem, p. 223. «Il Vittoriano, oltre a rappresentare il più grottesco esempio di spazio chiuso che ci viene in mente, è un'assurdità, remota nonostante la ciclopica mole incombente e soprattutto inaccessibile sotto tutti i punti di vista.» 


\section{Oculartux_saggi}

Ruggero Ragonese | Monument et espace urbain

$\mathrm{Si}$, par contre, en revenant à la première définition de De Sessa on entend par espace fermé un espace qui est séparé, on a, alors, la possibilité d'ébaucher un discours un peu plus intéressant.

L’Autel est un espace séparé, mais séparé de quoi? (N.d.T.) " Il peut être le résultat auquel vise un architecte qui, plus ou moins inconsciemment, refuse la réalité socio-culturelle, tend à extérioriser son rejet par des édifices qui sont à leur tour séparés du contexte". 3

L'Autel de la Patrie est donc un espace fermé, parce étranger à l'espace qui devrait l'englober (la ville de Rome). Le Vittoriano serait par conséquent un espace séparé de son contexte urbain, chose en partie vraie, mais qui mérite également un approfondissement.

L'Autel de la Patrie ne s'insère pas par erreur dans le tissu de la ville de Rome. Il suffit de consulter les chroniques parlementaires entre les années 1882 et 1885 , pour remarquer que chez tous les protagonistes de la création du monument, il y avait une conscience d'y être pour construire une œuvre architecturale qui n'était pas en harmonie avec son contexte. Une œuvre de 120 mètres de haut et 100 de large environ, construite entièrement en marbre blanc, ne peut certainement pas s'harmoniser avec une ville de structure médiévale, avec des édifices de modestes dimensions et construits pour la plupart en pierre. On ne pouvait pas non plus penser à une intégration de l'énorme masse du Vittoriano avec les restes des forums romains, étant donné que ces derniers sont en fait des ruines marquées par le temps, alors que ce qu'on allait construire était une espèce de fac-simile moderne des temps passés. Il ne s'agit donc pas d'une erreur ou d'un problème d'ignorance, mais d'un choix bien précis. Mais ceci est une banalité, relevée également par De Sessa: (N.d. T.) "Espace fermé, peut être le but vers lequel le régime totalitaire adresse, volontairement, ses expressions. "4

Le Vittoriano est donc un objet volontairement étranger au reste de la ville. Il est construit pour ne pas être confondu avec son contexte, pour rappeler quelque chose. Il est construit pour être reconnu. La notion d'espace, à ce niveau, ne nous suffit plus. Si définir le Vittoriano comme 'espace fermé ‘ parce qu'inaccessible était une assertion non confirmée par les faits, définir le Vittoriano comme espace fermé parce que séparé est insuffisant. Le Vittoriano n'est pas en fait séparé du reste de la ville, même s'il est au centre de cette dernière, mais plus simplement il est en contraste avec le reste de la ville. Il ne s'agit pas alors d'opposer un espace fermé (L'autel) à un espace éventuellement ouvert (la ville de Rome); Il s'agit, à notre avis, d'opposer plus simplement un espace vu comme homogène (la ville de Rome) à un espace vu comme non homogène et séparé (le Vittoriano). Séparé ne veut pas

3 Ibidem, p.207. «Può essere il risultato cui approda un architetto che rifiutando, più $o$ meno inconsciamente, la realtà socioculturale, tende ad estrinsecare questo suo rigetto con edifici a loro volta separati dal contesto.»

4 Ibidem, p.205. «Spazio chiuso può essere il fine verso cui si indirizza, volontariamente le sue espressioni il regime totalitario.» 


\section{Dcular $a_{\text {flux_saggi }}$}

Ruggero Ragonese | Monument et espace urbain

forcément dire fermé. Au contraire l'Autel de la patrie a continué pendant plus d'un siècle à ébaucher un dialogue avec l'espace qui l'entourait.

L'Autel de la patrie est donc un monument. Affirmation banale, c'est vrai, mais le monument n'est pas ici ce qui commémore un passé, mais c'est une récit de le passé. Alors qu'est-ce qu'on entend par Autel de la patrie en tant que monument? Simplement que notre objet s'est posé au centre d'une ville en la marquant de façon indélébile, créant une opposition comme nous avons vu, entre lui et le reste du tissu urbain (homogène et non homogène).

\section{Monumentaliser la ville}

«Monumentaliser au sens architectural, c'est par exemple transformer une porte en portail ou en portique; ou une simple chaise en prototype de chaise»: sans enlever sa fonction a un édifice ou un object, on veillera à la transcender par une mise en représentation da la chose par elle-même, qui s'autonomise ainsi de sa propre fonction. "Cette mise entre guillemets s'obtient en général par un double isolement dans l'espace. À la verticale, on exhausse (socle, piédestal, gradins ou pilotis à là Le Corbusier). À l'horizontale, on dégage (esplanade, perspective, terre-plein). Le monumental, c'est une masse mise en valeur par du vide» (Debray 1999: 35).

Le monumental est ce qui rompt le rythme de la ville, c'est ce qui crée le vide autour, au point de détruire tout ce qui l'entoure. Comme affirme justement Ragon "Le gigantisme ponctue toute l'histoire de l'architecture" (Ragon 1985: 74). Mais pourquoi cette nécessité de briser le rythme spatial qui difficilement s'est construit dans une ville? La réponse est dans la étymologie même du mot 'monument'. En effet, 'monument' vient du terme latin moneo, qui a son tour dérive du grec mnemeion, c'est-à-dire 'souvenir'. Le monument doit commémorer quelque chose ou quelqu'un. Mais construire un énorme monument, qui détruit en grande partie le tissu de la ville dans lequel il s'insère, ne veut pas seulement dire " rappeler", mais veut en quelque sorte: (N.d.T.) qu'un événement soit perçu comme important pour l'histoire; c'est-à-dire sémiotiquement marqué au niveau historique. C'est pourquoi du point de vue du présent on sélectionne et interprète les événements passés dans la mesure où dans la connaissance collective on en conserve le souvenir. Le passé est alors organisé comme un texte, lu du point de vue du présent. Le monument est alors à tous les effets un " appareil de transmission de l'espèce (...) Celui-ci communique une mise en perspective bien précise du passé; la mise en perspective la plus avantageuse bien entendu au pouvoir constitué (qu'il soit religieux, laïque, public ou privé). ” (Debray 1999: p.39) Le sujet institutionnel capable de financer, de choisir et d'imposer. La famille fait la demeure, l'Eglise fait l'église, l'entreprise fait l'usine; les pouvoirs publics font le monument public, solidaire qu'il est de l'espace public."

Le monument, en brisant l'ordre préétabli, dans un présent devenu passé, permet de remettre l'histoire passée dans une autre perspective: 


\section{Ocula ${ }^{\text {Flux_Saggi }}$}

Ruggero Ragonese | Monument et espace urbain

(N.d.T.) «L'expérience historique (c'est-à-dire une certaine interprétation du passé) a beaucoup d'influence sur le cours de l'histoire: c'est en fait à partir de telles représentations, d'une telle expérience que la société entendue comme personnalité collective construit son propre avenir, projette son propre comportement ultérieur.»5

Et pourtant celui qui (N.d.T.) " Regarde avec des yeux interrogatifs l'architecture, est pris par la sensation qu'elle est bien plus qu'un simple fait de communication de masse ". 6 L'objet architectural est un objet 'vécu', 'traversé' par les hommes. En effet l'espace du monument non seulement communique, mais est capable également de "stipuler une cérémonie, soutenir un rituel, interpeller une postérité " (Debray 1999: 311). Il ne se présente pas seulement comme pur objet, comme instrument pour communiquer, mais également comme lieu à utiliser. On a alors deux points d'analyse: d'une part, on peut étudier la structure de l'objet architectural. On peut observer le monument en lui-même. D'autre part, on peut regarder les parcours qui se sont dénoués à l'intérieur de ce monument. Les routes doivent être toutes les deux entreprises. Non pas comme deux routes parallèles, mais au contraire comme deux routes qui ensemble se dévoilent et se soutiennent. Hamon affirme que présupposer (N.d.T.) «la vie en commun entre le récit, ou le procès de configuration du temps à travers un système de signes discrets, distinctifs, linguistiques, et l'architecture processus de mesure et de configuration de l'espace à travers limites et démarcations de différents types» 7 est une hypothèse qui attend des réalisations pratiques. Le récit et l'architecture sont

(N.d.T.) «Deux structures de domination du monde, deux rythmisations du monde obtenues 'en donnant au rythme' le sens (structure, distribution formelle) qui lui appartient étymologiquement, comme nous rappelle Benveniste dans son Problèmes de linguistique générale. Le Discours dévoile les structures de l'Objet. L’Objet fonde et permet le discours. Par conséquent, les déplacements des hommes ne jouent alors qu'un rôle révélateur, et la hypervalorisation de certaines directions du référentiel architectural dépend en fait des directions cardinales qui prennent de la valeur d'un système cosmogonique transcendant. Cette terminologie est conforme à "la tradition sémiotique qui distingue l'univers immanent de la narration et l'univers trascendant des Destinateurs.» 8

5 Uspenskij (1988), p. 15. «A sua volta, l'esperienza storica (cioé una certa interpretazione del passato) influisce natrualmente sula corso futuro della storia: è infatti a partire da simili rappresentazioni, da una simile esperienza, che la società come personalità collettiva costruisce il programma del futuro, progetta il suo comportamento ulteriore.»

610 Eco (1968), p.229. «Eppure chi guarda con occhio interrogativo l'architettura, è colto dalla sensazione che essa sia qualcosa in più di un fatto di comunicazione di massa.»

7 Hamon (1996), p. 25. «la convivenza fra il racconto, ovvero il processo di configurazione del tempo attraverso un sistema di segni discreti, distintivi, linguistici, e l'architettura, processo di misurazione e configurazione dello spazio mediante tramezzi e demarcazioni di vario tipo.»

8 Hammad, (1988), p.243. "Due strutture di dominio del mondo, due ritmizzazioni del mondo, ottenute 'dando al ritmo' il senso (struttura, distribuzione formale) che è etimologicamente suo, come ci ricorda opportunamente Benveniste nel suo Problemi di Linguistica generale. Il Discorso svela le strutture dell'Oggetto. L’Oggetto fonda e rende possibile il Discorso. Di conseguenza gli spostamenti degli uomini non giocano altro che un ruolo rivelatore, e la supervalorizzazione di certe direzioni del referenziale architettonico dipende di fatto dalle direzioni cardinali che prendono valore da un sistema cosmogonico trascendente. Questa terminologia è 


\section{Dcular $a_{\text {flux_saggi }}$}

Ruggero Ragonese | Monument et espace urbain

En 1982, Alain Renier se rend compte de la nécessité de faire passer l'étude de l'architecture dans une nouvelle considération de sa dimension historique, «au risque de reconstituer le microcosme culturel dont une partie des architectes avait réussi à s'extraire.» (Renier 1982: 12)

Il s'agit enfin d'éclairer le problème lié à la physicité de l'objet architectural. La sémiotique de l'architecture est donc la discipline, "qui étudie l'architecture en tant que système de signification; elle ne nie pas que l'architecture soit fortement déterminée par son système de 'construction' physique, mais elle postule que celui-ci ne peut exister qu'en étroite liaison avec le système de signification.» (Ibidem: 13)

On aura alors une sémiotique de l'architecture qui interroge le système de manifestation qui résulte des faits de production de l'espace et des transformations opérées par les multiples d'usage de la vie quotidienne.

\section{Petite conclusion sur semiotique et architecture}

Il existe par conséquent une sémiotique de l'architecture autonome qui interroge un système de manifestation spécifique (faits de production et des faits d'usage). Mais cette sémiotique autonome n'est pas indépendante parce que corrélée à de nombreuses sémiotiques particulières qui se constituent en d'autres domaines de connaissances. Les objets architecturaux ne peuvent pas être considérés tous seuls mais doivent être encadrés à travers de nombreuses pratiques scripturaires, graphiques et plastiques. La sémiotique de l'architecture peut ainsi parfaitement se raccorder à cette base théorique que nous avons essayé de tracer dans les grandes lignes au cours de l'introduction. peut pas être vu comme scindé de son Discours: textes, simulacres et calculs dont " l'existence n'est pas dissociable du produit final, l'ouvrage réalisé, transformé par l'usage en une œuvre incessante." (Ibidem: 12)

C'est justement l'importance de la dimension historique située dans l'espace architectural à rendre prééminent une analyse sémiotique qui fasse référence à chaque fois aux divers textes et discours, manifestés dans l'usage de l'espace, et justifiable ainsi chacun d'une étude sémiotique.

La possibilité d'effectuer une analyse de sémiotique de l'architecture part de la considération que l'objet

architectural se base en son déchiffrement, en son parcours. La ville, les monuments ne sont pas des textes en soi; les parcours à l'intérieur de la ville les constituent comme texte. Le texte urbain n'existe pas sans ses lectures. Ces lectures ne sont pas immédiatement données par la ville ou par le monument, mais sont discernables dans les textes.

Ceci ne veut pas dire que nous sommes en contradiction avec ce que nous avons soutenu en introduction, que l'objet architectural en soi n'est pas ana-

conforme alla tradizione semiotica che distingue tra l'universo immanente della narrativa e l'universo trascendente dei Destinatori.» 


\section{Oculartax_saggi}

Ruggero Ragonese | Monument et espace urbain

lysable; Il s'agit tout simplement d'adopter «deux attitudes en la matière: soit 'photographier' du regard et transformer ainsi l'objet plastique en une image instantanée, soit parcourir des yeux l'œuvre plastique et y inscrire un itinéraire.» (Ibidem: 18)

Il reste une question à poser: est-il juste de se limiter à tracer un parcours? À notre avis, non. L'analyse sémiotique doit enquêter sur des discours qui ont en quelque sorte représenté le lieu donné ou le monument donné, doit à travers l'analyse de ces derniers être capable de reconstruire une histoire du lieu, du monument: une histoire faite de parcours. Les parcours décrivent en définitive un parcours unique, celui du monument vu dans ses stades successifs, dans ses 'cristallisations'.

Notre proposition est, par conséquent, celle d'une histoire du regard: une série de 'visions' sur le monument. Il pourrait y en avoir beaucoup d'autres: on peut voir le monument dans ses différentes représentations photographiques, dans ses images picturales. On aurait pu également observer comment le monument s'insère dans les cartes de la ville. Pour décrire le caractère visuel d'un édifice, par exemple, nous pouvons mettre en lumière les différents modes d'utilisations des données iconiques au service de l'architecture, en tentant de répondre notamment à la question des vastes ensembles de données iconiques constitués en corpus représentatifs d'objets architecturaux et urbains, réels ou projetés: «afin de dépasser leur statut habituel et réducteur d'illustration pour mettre au jour leur contenu d'information et de signification» (Sanson, 1998). Tous ces parcours, en fait, dévoilent et ouvrent le texte architectural vers une analyse plus précise qui révèle la trace d'un passé rémanent et la structure d'un avenir à faire. Entre boucle récursive et mise en abîme, la confrontation de ces représentations ouvre des perspectives théoriques et pratiques plus ambitieuses. Ce travail exploratoire constitue l'étape préalable à des recherches articulant culture architecturale et information documentaire. La perception de la ville s'organise lors de l'appropriation 'cursive' de celle-ci par ses habitants. En se construisant, la perception opère son travail de filtrage. Ainsi, tout bâtiment ou tout lieu urbain peuvent être considérés comme un élément d'une chaîne d'espaces parcourus successivement. Plus encore, la ville est lieu de multiples parcours et chacun de ses éléments s'inscrit non seulement dans une successivité spatiale, mais dans une réseau de connexité constitué par plusieurs textes et paysages urbains. 


\section{Ocula ${ }^{\text {Fux__aggi }}$}

Ruggero Ragonese | Monument et espace urbain

\section{Bibliographié}

Debray, R.

1999 “Trace, forme ou message?", dans Les Cahiers de médiologie n. 7, Paris, Gallimard, pp. 27-47.

De Sessa, C.

1990, Capire lo spazio architettonico, Roma, Officina edizioni.

Eco, U.

1968 La struttura assente, Milano, Bompiani (La structure absente, traduit par Uccio Esposito Torrigiani, Paris, Mercure de France, 1972).

Hammad, M.

1988 “Tre sistemi sono necessari...”, dans Carte semiotiche 4-5, Firenze, La casa Usher.

Hamon, P.

1996 "La finestra " dans Testo letterario e immaginario architettonico, dir. CASARI, R., LORANDI, M. , PERSI, U., RODRIGUEZ AMAYA, F., Milano, Jaca book, pp. 25-30.

RAGON, M.

1985 “Architecture et mégastructures ”, dans Communications 42, Seuil, pp. 6979.

Renier, A.

1982 "L'espace e la représentation comme objets sémiotiques", dans Espace et architecture, Paris, La Villette.

Sanson P.

1998 "Histoire des données iconiques relatives à l'espace habité", Solaris nº4, [en ligne]

http://biblio-fr.info.unicaen.fr/bnum/jelec/Solaris/do4/4sanson.html

USPENSKIJ, B., 1988, Storia e Semiotica, Milano, Bompiani. 